\title{
Understanding Unsteady Flow Physics of Two Types of Vertical Axis Wind Turbines with a Fluid-Structure Interaction Approach
}

\author{
Kan Liu ${ }^{1}$, Meilin $\mathrm{Yu}^{2}(\bowtie)$, and Weidong $\mathrm{Zhu}^{3}$ \\ Department of Mechanical Engineering \\ University of Maryland, Baltimore County (UMBC), Baltimore, MD 21250
}

Unsteady flow physics of two types of vertical axis wind turbines (VAWTs), namely, a modified Savonius turbine and a hybrid Darrieus-modified-Savonius (HDMS) turbine, are numerically studied using a fluid-structure interaction approach. As a first step, a numerical solver is developed by coupling the wind turbine dynamics with a high-fidelity flow solver, thus, synchronizing the flow-turbine interaction. The solver is then used to study unsteady aerodynamics of wind-driven modified Savonius and HDMS VAWTs under different loading conditions. The relationship between the power efficiency and tip speed ratio (TSR) is invested for both types of wind turbines. It is found that there exists large disparity on energy harvesting performance between the two types of VAWTs under wind-driven flow conditions. The modified Savonius VAWT achieves relatively high power efficiency $(\sim 30 \%$ or $\sim 51 \%$ of the Betz's limit) at small TSRs; the HDMS VAWT achieves high power efficiency $(\mathbf{4 0 \%}$ or $\sim 67 \%$ of the Betz's limit) at large TSRs. At the same incoming wind speed, the maximum power efficiency of the HDMS VAWT is about $10 \%$ higher than that of the Savonius VAWT. It is also observed that the external loading can affect dynamic stall over Darrieus blades of the HDMS VAWT, thus significantly varying the TSR and the corresponding energy harvesting efficiency.

\section{Nomenclature}

$\begin{array}{ll}C & =\text { damping factor } \\ I & =\text { rotational inertia } \\ \dot{\theta} & =\text { angular velocity } \\ \ddot{\theta} & =\text { angular acceleration } \\ M & =\text { moment } \\ C_{m} & =\text { moment coefficient } \\ \rho & =\text { air density } \\ V & =\text { wind velocity } \\ \tau & =\text { non-dimensional time step } \\ L & =\text { chord length } \\ A & =\text { wind swept area } \\ P & =\text { power extracted by wind turbines } \\ P_{\max } & =\text { total wind power } \\ C_{p} & =\text { power coefficient }\end{array}$

\footnotetext{
${ }^{1}$ Graduate Student, Department of Mechanical Engineering, email: kan7@umbc.edu

${ }^{2}$ Assistant Professor, Department of Mechanical Engineering, AIAA Senior Member, email: mlyu@umbc.edu

${ }^{3}$ Professor, Department of Mechanical Engineering, email: wzhu@umbc.edu
} 


\section{Introduction}

Due to the depletion of fossil energy and pollution aggravation, there is an imperious demand of reliable energy harvesting methods with low carbon emission. Wind energy is a green and renewable energy source which has been utilized globally nowadays. There are dominantly two types of wind turbines, namely, horizontal axis wind turbines (HAWTs), and vertical axis wind turbines (VAWTs), for wind energy harvesting. Currently, HAWTs have been adopted in majority of utility-scale applications, e.g., wind farms. However, comparing with HAWTs, VAWTs have several advantages [1], including: (1) VAWTs are insensitive to wind directions, and can harvest energy from wind coming in any direction; (2) VAWTs' rotor and blades have longer operating life than HAWTs since they only encounter constant gravity and inertia loads in terms of quantity and direction; (3) VAWTs can be installed close to the ground, on a rooftop or in an urban area where wind is more turbulent than that in an open wind farm; and (4) VAWTs are typically quieter than HAWTs, thus leading to less acoustic pollution. However, several challenges in the VAWT design, including poor self-starting capability, low power harvesting efficiency, and structural resonance and fatigue under complex aerodynamics loads, should be tackled before VAWTs can be mass-produced to enhance wind energy production.

There are currently two dominant designs for VAWTs, namely Darrieus and Savonius designs. Several recent comprehensive reviews of the two designs can be found in Refs. [2, 3, 4]. The Darrieus VAWT, is a lift-driven wind turbine, and usually has high energy harvesting efficiency at relatively large tip speed ratios (TSRs). But it suffers from self-starting issues due to the dead band of negative torque at small TSRs $[5,6,7]$. The Savonius VAWT is self-starting, and works well at small TSRs [8, 9, 10]. But the energy harvesting efficiency is much lower than that of the Darrieus VAWT. In this study, to overcome the deficiencies of both individual turbine designs, these two types of VAWTs are combined to a hybrid Darrieus-modified-Savonius (HDMS) one by placing the Savonius rotor in the central region of the Darrieus rotor. We note that several works on the combined Darrieus and Savonius designs with different hybrid configurations have been reported in literatures $[11,12,13]$. The superior aerodynamic performance of the new HDMS design will be demonstrated by comparing with that of a modified Savonius design.

Experiments have demonstrated that the wakes behind motor-driven and wind-driven wind turbines have similar vortical structures, as long as the rotational motion is similar [14]. As a matter of fact, several researchers have numerically studied wake structures by prescribing VAWTs' motion at a constant angular velocity $[15,1]$. However, for experimental studies, researchers usually prefer using VAWTs driven by wind [12]. Note that numerical simulation of VAWTs with prescribed motion cannot account for flow physics during the starting stage of wind turbines. To investigate the self-starting issue, especially for Darrieus VAWTs, numerical simulation of wind-driven VAWTs still needs to be performed [16]. In the current study, numerical simulation is conducted for the wind-driven modified Savonius and HDMS VAWTs to investigate both the self-starting capability and the power efficiency of these turbines.

The remainder of this paper is organized as follows. In Section II, numerical methods and simulation setup are introduced. In Section III, the self-starting capability and power efficiency of both the modified Savonius and HDMS VAWTs with the same Reynolds number are numerically studied under wind-driven conditions. Different damping factors are used to reach different final TSRs. Numerical results from each type of VAWTs are presented and discussed there. Section IV briefly concludes the study and discusses the future work. 


\section{Numerical methods}

\section{II.A. Governing equations}

The governing equations of fluid flow are unsteady Reynolds-averaged Navier-Stokes (URANS) equations. In this study, the turbulence model is selected as the Spalart-Allmaras (S-A) model. Thus, the governing equations can be written as

$$
\left\{\begin{array}{l}
\frac{\partial u_{j}}{\partial x_{j}}=0 \\
\frac{\partial u_{i}}{\partial t}+\frac{\partial u_{i} u_{j}}{\partial x_{j}}=-\frac{1}{\rho} \frac{\partial p}{\partial x_{i}}+\frac{\partial}{\partial x_{j}}\left[v\left(\frac{\partial u_{i}}{\partial x_{j}}+\frac{\partial u_{j}}{\partial x_{i}}-\frac{2}{3} \delta_{i j} \frac{\partial u_{l}}{\partial x_{l}}\right)\right]+\frac{\partial}{\partial x_{j}}\left(-\overline{\bar{u}_{i}^{\prime} u_{j}^{\prime}}\right) \\
\frac{\partial \tilde{v}}{\partial t}+\frac{\partial \tilde{v} u_{j}}{\partial x_{j}}=\frac{1}{\sigma_{\tilde{v}}}\left[\frac{\partial}{\partial x_{j}}(v+\tilde{v}) \frac{\partial \tilde{v}}{\partial x_{j}}+C_{b 2} \frac{\partial \tilde{v}}{\partial x_{j}} \frac{\partial \tilde{v}}{\partial x_{j}}\right]+G_{v}-Y_{v}
\end{array}\right.
$$

where $\rho$ is the density (constant for incompressible flow), $u_{i}$ is the velocity in the $i$ th direction, $p$ is the pressure, $\tau_{i j}$ is the stress tensor, $\tilde{v}$ is the turbulence working variable in the SA model, $v$ is the kinematic viscosity of the fluid, $G_{v}$ is the production of turbulent viscosity, $Y_{v}$ is the destruction of turbulent viscosity, and $\sigma_{\widetilde{v}}$ and $C_{b 2}$ are model constants. Note that the Einstein summation convention is used in Eq. (1) to achieve notational brevity.

In this study, the governing equation is solved using the ANSYS $®$ Fluent ${ }^{\circledR} 15.0$ commercial CFD software. Specifically, the second-order pressure-velocity coupling scheme is selected for spatial discretization. The second-order implicit time discretization is used to advance the unsteady simulation in the time direction. The sliding mesh model is used to accommodate the grid motion. To achieve the wind-driven simulation, the flow solver is then coupled with the kinematics equation as specified in the User Defined Functions (UDFs).

\section{II.B. Wind turbine kinematics and power coefficient calculation}

Assume that the VAWT is rigid, and only rotates with respect to its central axis. The governing equation of wind turbine kinematics is expressed as follows

$$
I \ddot{\theta}=M_{\text {wind }}-M_{\text {load }},
$$

where $I$ is the rotational inertia, $\ddot{\theta}$ is the angular acceleration, $M_{\text {wind }}$ is the aerodynamic moment, and $M_{\text {load }}$ is an external load. For practical wind engineering application, the external load can be an intricate function of the angular velocity $\dot{\theta}$ or acceleration $\ddot{\theta}$ of the turbine. In this study, a linear relationship between $M_{\text {load }}$ and $\dot{\theta}$ is assumed, namely, $M_{\text {load }}=C \dot{\theta}$, where $C$ is the damping factor. As a result, Eq. (2) can be rewritten as

$$
I \ddot{\theta}+C \dot{\theta}=M_{\text {wind }} .
$$

Note that in the ANSYS ${ }^{\circledR}$ Fluent ${ }^{\circledR}$ software, $M_{\text {wind }}$ is calculated from the flow solver as $M_{\text {wind }}=$ $\frac{1}{2} C_{m} \rho U_{\infty}^{2} A L$, where $C_{m}$ is the moment coefficient, $U_{\infty}$ is the free stream velocity, A is the reference area, and $\mathrm{L}$ is the reference length.

After specifying the governing equations of the fluids and the rigid-body motion of the turbine, the fluid-structure interaction approach can be developed as follows. When Eq. (1) is solved, the aerodynamic moment $M_{\text {wind }}$ in Eq. (2) is available. The angular displacement $\theta$ of the VAWT can then 
be calculated from Eq. (2). This will provide new boundary conditions for Eq. (1), which will be solved again to obtain new aerodynamic moment. This completes the coupling between fluid flow and wind turbine kinematics.

After obtaining the aerodynamic moment and angular velocity, the power extracted from wind is calculate as follows:

$$
P=M_{\text {wind }} \dot{\theta}
$$

Note that the total power in the incoming wind passing across the turbine with a reference area A can be calculated using the following formula

$$
P_{\max }=\frac{1}{2} \rho U_{\infty}^{3} A
$$

Finally, the power efficiency $C_{\text {power }}$ is calculated as:

$$
C_{\text {power }}=\frac{P}{P_{\max }} \text {. }
$$

Based on the Betz's law, the maximum value of the power efficiency $C_{\text {power }}$ is $59.3 \%$ in an open flow [17].

\section{II.C. Configuration of wind turbines, meshes and simulation setup.}

\section{II.C.1. Two types of VAWTs}

As mentioned previously, two types of wind turbines are simulated in this study. One is the modified Savonius type, and the other is the HDMS type. 3D models of the two types of VAWTs are displayed in Figure 1. In this study, 2D X-Y plane profiles of the two VAWTs, are presented in Figure 2, are used in numerical simulation. The meshes around the two VAWTs are shown in Figure 3. Due to that the second-order accurate numerical methods are used in this simulation, high-quality meshes are required around the Darrieus blades in the HDMS VAWTs to capture the intricate vortex dynamics. The mesh refinement will be discussed in Section II.C.2. It has been verified that the averaged $Y^{+}$in numerical simulation is smaller than one.

The two VAWTs have the same radius of $0.7 \mathrm{~m}$. Other important parameters in numerical simulation are documented in Table 1.
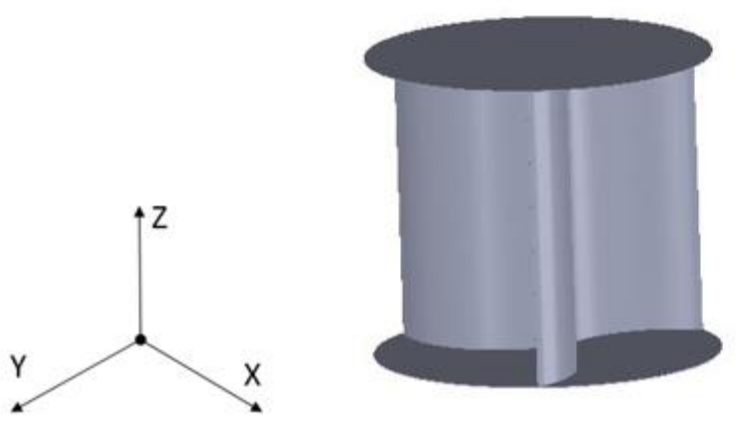

(a)

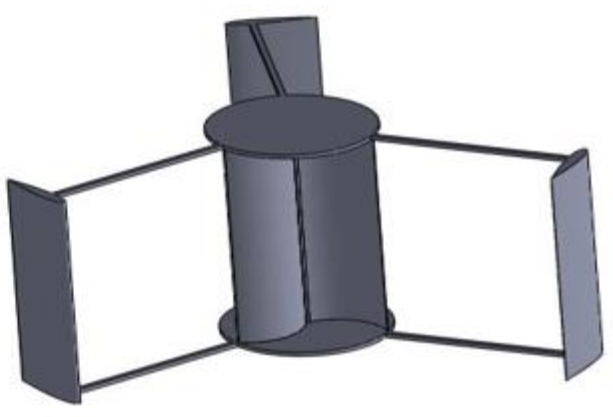

(b)

Figure 1. 3D profiles of (a) Savonius VAWTs; and (b) HDMS VAWT. 


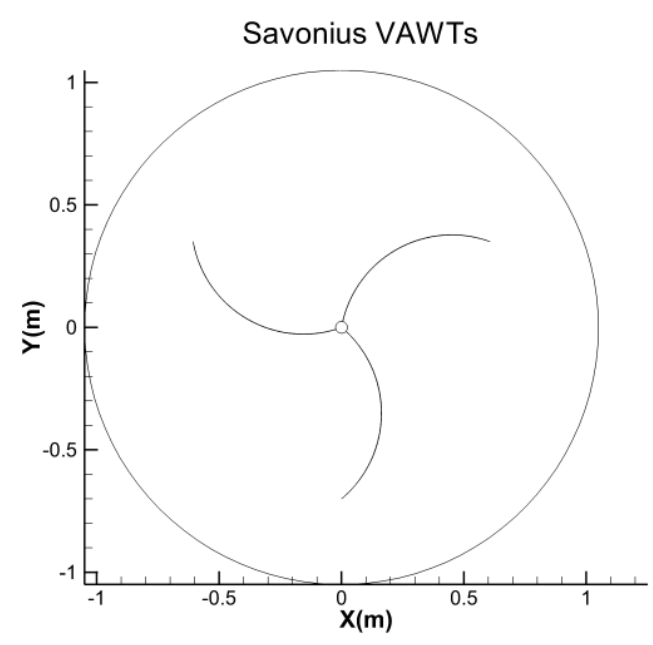

(a)

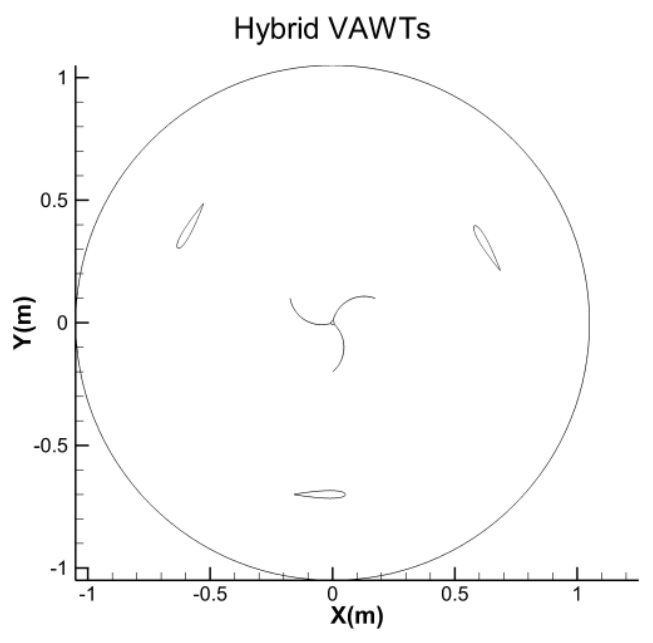

(b)

Figure 2. (a) Shape of the modified Savonius VAWTs; (b) Shape of the HDMS VAWTs.

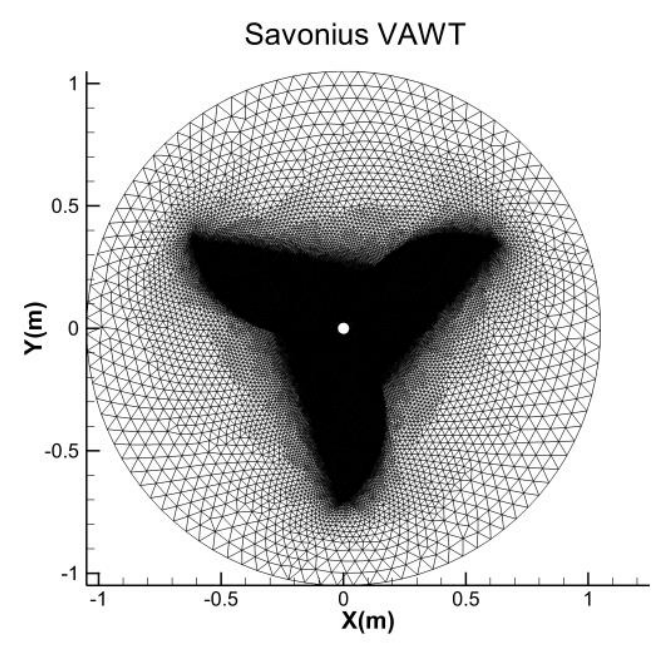

(a)

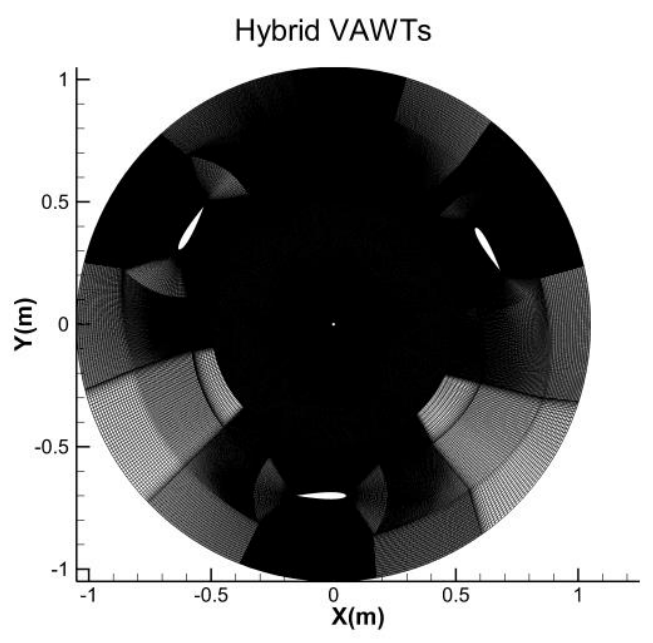

(b)

Figure 3. (a) Mesh of the modified Savonius VAWTs; (b) Mesh of the HDMS VAWTs.

\section{II.C.2. Mesh refinement study and benchmark test}

To estimate the accuracy of the mesh used in this study, a benchmark test had been simulated. Li et al. [1] presented the relationship between $C_{p}$ and TSR of Darrieus VAWTs with different methods. Therefore, the Darrieus part, as shown in Figure 4, of the HDMS VAWT is used. For the purpose to maintain the same Reynold number, a geometry as twice large as the one shown above is generated. 
Table 1. Specifications of the two types of VAWTs

\begin{tabular}{|c|c|c|}
\hline VAWTs & $\begin{array}{c}\text { Modified } \\
\text { Savonius }\end{array}$ & HDMS \\
\hline $\begin{array}{c}\text { Nominal wind } \\
\text { speed (m) }\end{array}$ & 5 & 5 \\
\hline Total radius (m) & 0.7 & 0.7 \\
\hline $\begin{array}{c}\text { Outer blades } \\
\text { shape }\end{array}$ & N/A & NACA0015 \\
\hline $\begin{array}{c}\text { Outer blades } \\
\text { chord length(m) }\end{array}$ & N/A & 0.21 \\
\hline Inner blades radius & N/A & 0.2 \\
\hline Swept area (m $\left.{ }^{2}\right)$ & 1.4 & 1.4 \\
\hline Reynolds number & $4.9 \times 10^{5}$ & $4.9 \times 10^{5}$ \\
\hline
\end{tabular}

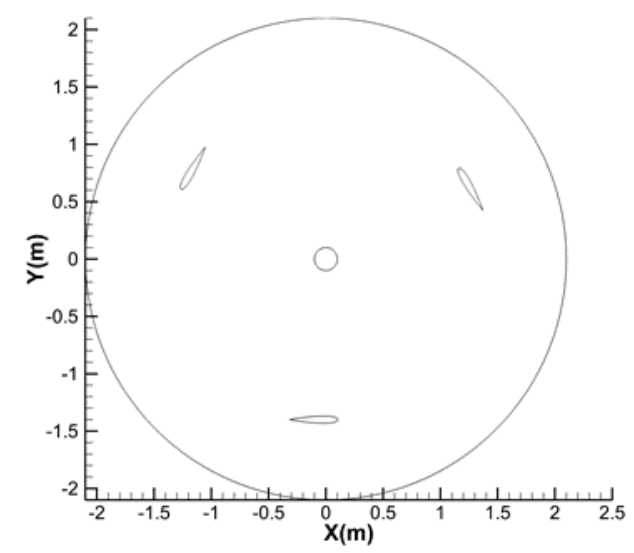

Figure 4. Darrieus VAWT used in the benchmark test.

Additionally, three mesh are used in this simulation, named coarse mesh (104823 elements), medium mesh (433939 elements), fine mesh (1164156 elements) respectively. The refinement is carried out in both the radial and circumferential directions. The medium mesh has double the number of elements in both directions comparing with the coarse one. In the same way, the fine mesh double the number of elements of the medium one (see in Figure 5).
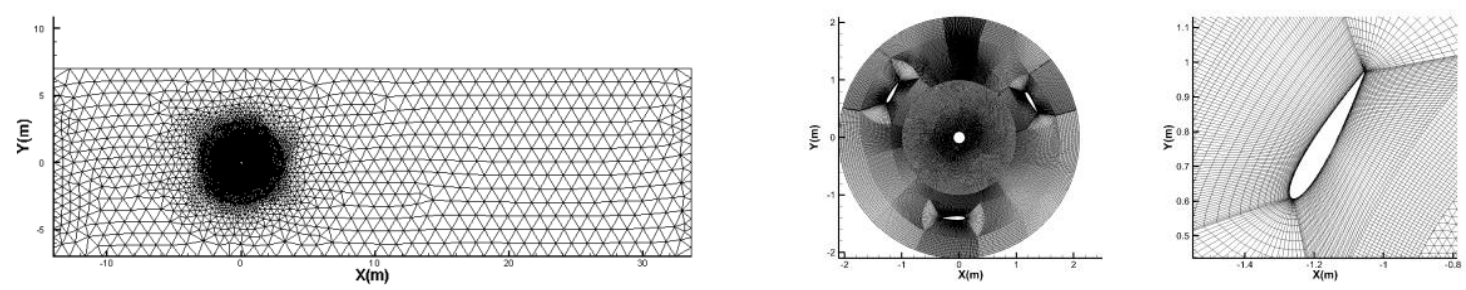

(a) Coarse mesh 

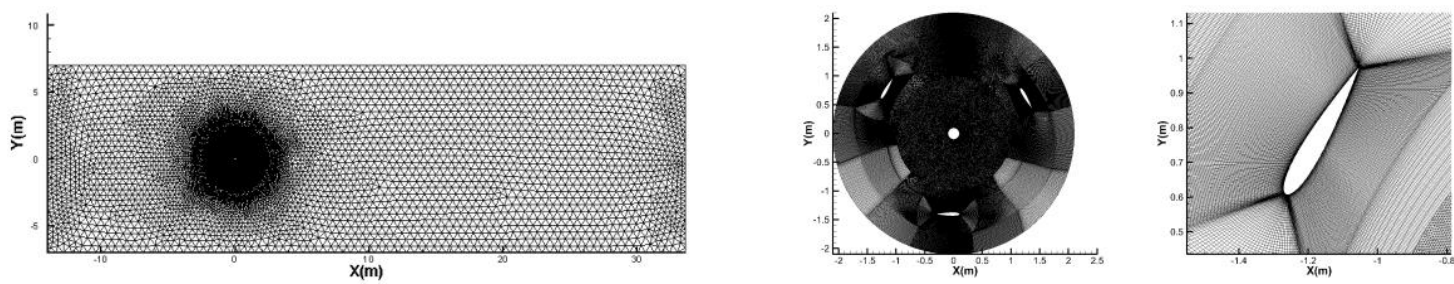

(b) Medium mesh
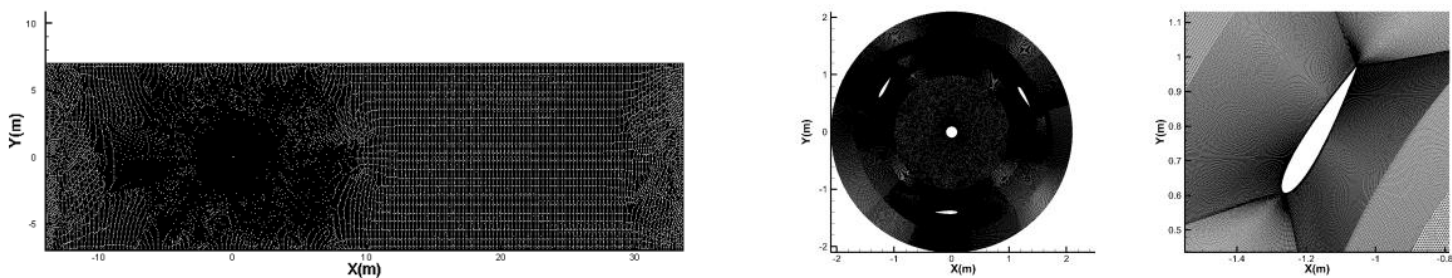

(c) Fine mesh

Figure 5. Three meshes used in the benchmark test.

With the same Reynold number, the results of the benchmark test are shown in Figure 6. It can be seen that the results of the three meshes not only predict the variation trend of $C_{p}$, but also was in reasonable agreement with magnitude of the wind tunnel result obtained by McLaren [18]. Meanwhile, the difference between the medium mesh and the fine mesh is less than $10 \%$. Therefore, to save computational cost, the medium mesh is selected for the simulations in this study.

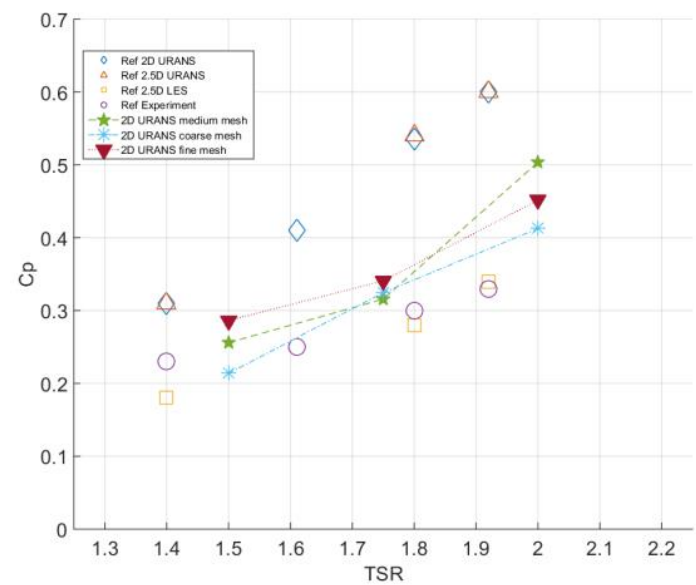

Figure 6. TSRs and power coefficients from the benchmark tests using three meshes and those from previous numerical and experimental results $[18,1]$.

\section{II.C.3. Time step refinement study}

For transient simulation of the highly unsteady flow passing over the VAWTs, flow physics can be sensitive to the time step. Sørensen et al. [19] and Travin et al. [20] suggested that the non-dimensional time steps $\tau=\Delta t \cdot V / L$ could be about 0.01 and 0.025 . To study the time step, and simulation in the next section, the medium mesh shown above is used and the wind velocity $V$ is set to $10 \mathrm{~m} / \mathrm{s}$. The whole test is simulated by prescribed motion of TSR $=1.5$. The moment coefficient of different time step is shown in Figure 7 and Table 2. 


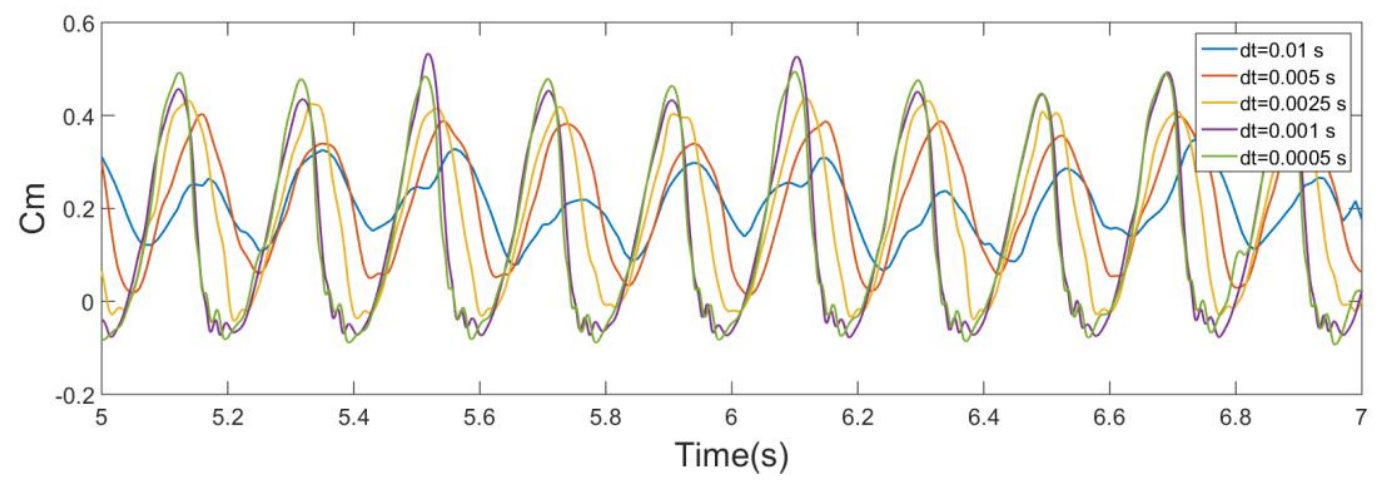

Figure 7. Moment coefficient of prescribed motion when $V=10 \mathrm{~m} / \mathrm{s}$ and $\mathrm{TSR}=1.5$.

Table 2. Time step and mean moment coefficient

\begin{tabular}{|c|c|c|c|c|c|}
\hline Time step (s) & 0.01 & 0.005 & 0.0025 & 0.001 & 0.0005 \\
\hline Mean $C_{m}$ & 0.1993 & 0.2139 & 0.1884 & 0.1475 & 0.1472 \\
\hline
\end{tabular}

From Figure 7, it can be seen that when $\mathrm{dt}=0.001 \mathrm{~s}(\tau=0.025)$ and $\mathrm{dt}=0.0005 \mathrm{~s}(\tau=0.0125)$, the difference between the histories of $C_{m}$ is small. It is also found that the difference of the mean $C_{m}$ between the two cases with different $\mathrm{dt}$ are less than 1\%. To save computational cost, $\mathrm{dt}=0.001 \mathrm{~s}$ is selected for the simulations below.

\section{II.C.4. Simulation setup}

In all simulation presented in this study, the following strategies are adopted to set up initial conditions. As a first step, the damping factor $\mathrm{C}$ is set to zero. This indicates that at the starting stage, there is no damping force acting on the VAWT. When the angular velocity of the VAWT achieves a stable (e.g., periodic) value, the damping effect will be added to the turbine. Note that when there is no damping effect, no wind energy will be extracted from the blowing wind (except the energy collected to accelerate the VAWT during the startup stage). When the damping effect (i.e., energy harvesting mechanism) is added to the VAWT, the aerodynamic torque is generated to overcome the resistance originated from the energy harvesting mechanism. As a result, the turbine system starts to collect energy from the wind.

\section{Results and discussions}

\section{III.A. Self-starting capability of two types of VAWTs}

Dominy et al. [21] reported that the three-bladed Darrieus VAWTs are able to rotate at a relatively low wind speed. In the current study, self-starting capability of VAWTs is defined as that the wind turbines should reach the desirable TSRs, at which the turbines can effectively harvest wind energy. It is observed from Eq. (3) that large inertia of VAWTs would lead to small acceleration. As has been recognized, Darrieus VAWTs at low angular velocity would encounter large dynamic stall [16], which can significantly decrease the aerodynamic moment acting on the turbines. Hence, it is necessary to optimize the inertia of VAWTs which can lead to the desirable TSRs.

To study the effects of inertia on the start-up process of VAWTs, simulation is carried out for both HDMS and modified Savonius VAWTs in free loading circumstance. The relationship between inertia and the angular velocity history of HDMS VAWTs is shown in Figure 8. It is observed that for HDMS 
VAWTs, only when the inertia is relatively small $\left(\mathrm{I}=0.1 \mathrm{~kg} \cdot \mathrm{m}^{2}\right)$, the angular velocity can reach a high value of about $27 \mathrm{rad} / \mathrm{s}$. In the Figure 8 (b) and (c), it is clearly seen that there exist a large-scale vortex shedding (or flow separation) around VAWTs when $I=1 \mathrm{~kg} \cdot \mathrm{m}^{2}$, resulting in dynamic stall over the Darrieus blades; almost no flow separation occurs when $I=0.1 \mathrm{~kg} \cdot \mathrm{m}^{2}$. This phenomenon would be further analyzed in the Section III.C. For the modified Savonius VAWTs, it is found that the final angular velocity almost does not depend on the inertia, but other parameters, e.g, damping factor, and wind speed.

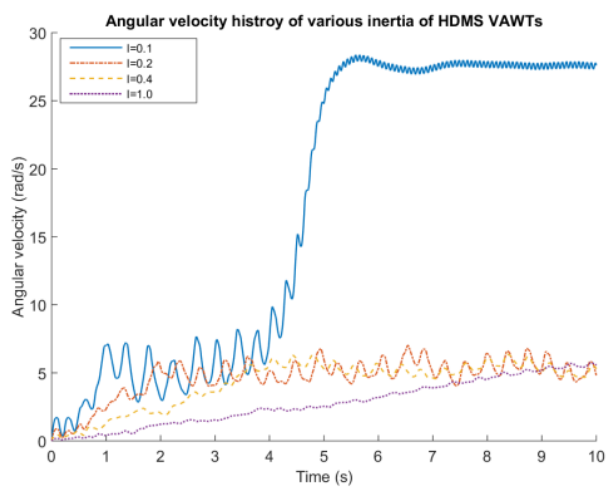

(a)

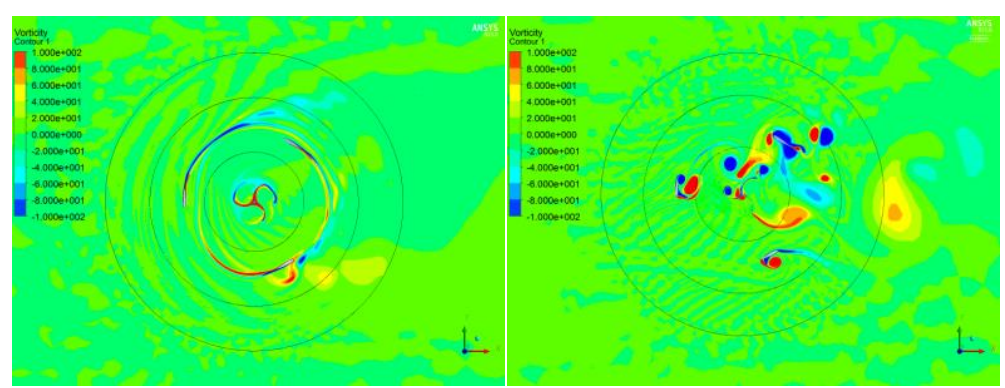

(b)

(b)

Figure 8. (a) Histories of the angular velocity for the HDMS VAWTs with $\mathrm{C}=0$; (b) vorticity field when $I=$ $0.1 \mathrm{~kg} \cdot \mathrm{m}^{2}$; (c) vorticity field when $I=1 \mathrm{~kg} \cdot \mathrm{m}^{2}$.

\section{III.B. Modified Savonius VAWTs}

As shown in Figure 9 (a), when the rotation of the modified Savonius VAWT becomes stable, the aerodynamic moment is periodic. In Figure 9 (b) and (c), pressure fields at two phases where the maximum and minimum aerodynamic moment values occur are presented as well. It is observed from Figure 9 (b) that when the wind moment reaches its maximum value, the pressure difference between the two surfaces of blade $\mathrm{A}$ is large, which drives it to move in the anti-clockwise direction. In contrast, it is observed from Figure 9 (c) that there exists a large pressure difference on the tip region of the blade $\mathrm{B}$, which can hinder the rotation of the Savonius turbine. This severely decreases the total aerodynamic moment in the anti-clockwise direction, thus degrading the energy harvesting efficiency.

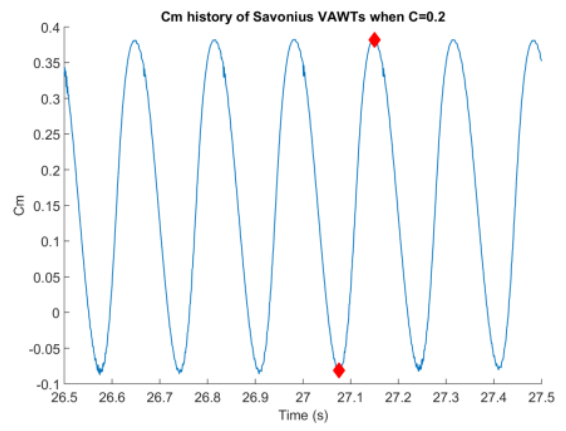

(a)

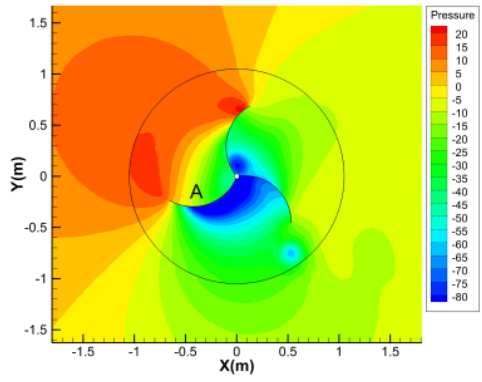

(b)

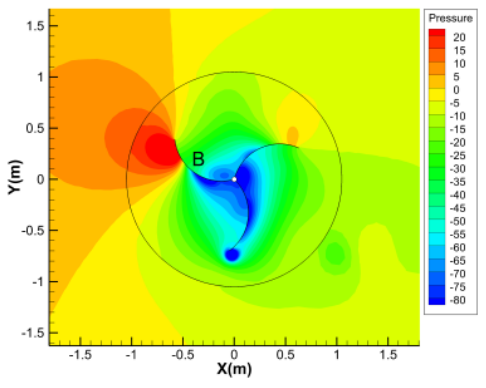

(c)

Figure 9. (a) $C_{m}$ history; (b) Pressure field corresponding to the maximum $C_{m}$ value; and (c) pressure field corresponding to the minimum $\mathrm{C}_{\mathrm{m}}$ value. 
For the modified Savonius VAWT, when the damping factor $\mathrm{C}$ increases, a smaller final angular velocity will be achieved, as shown in Figure 10 (a). In Figure 11 (a), both power coefficient and aerodynamic moment are displayed as a function of TSRs. It is observed that, for the modified Savonius VAWT, the aerodynamic moment increases when the TSR decreases. However, there exists an optimal total power output when the TSR is approaching 1.2. From Figure 11 (a), it is also observed that the maximum power efficiency of the modified Savonius VAWTs is about 28\%, which is about half of the theoretical maximum value $(59.3 \%)$ based on the Betz's law [17]. The variation patterns of both the power efficiency and moment coefficient have reasonable agreement with the experimental results obtained by Wekesa et al. [22] using a three-bladed Savonius VAWT.

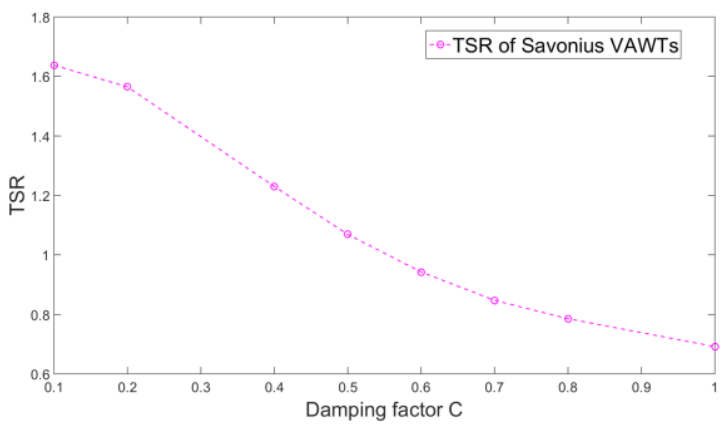

(a)

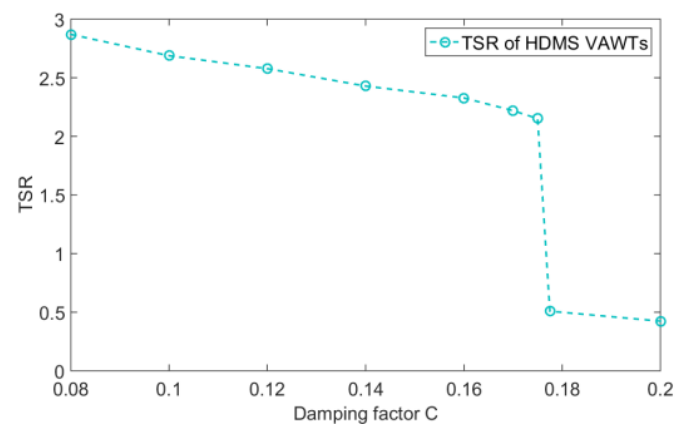

(b)

Figure 10. TSRs as a function of damping factors for (a) the Savonius VAWT and (b) the HDMS VAWT

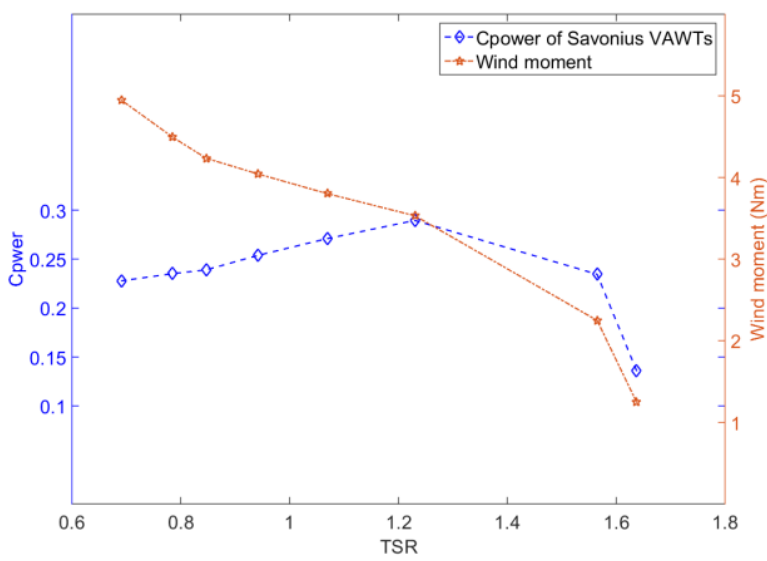

(a)

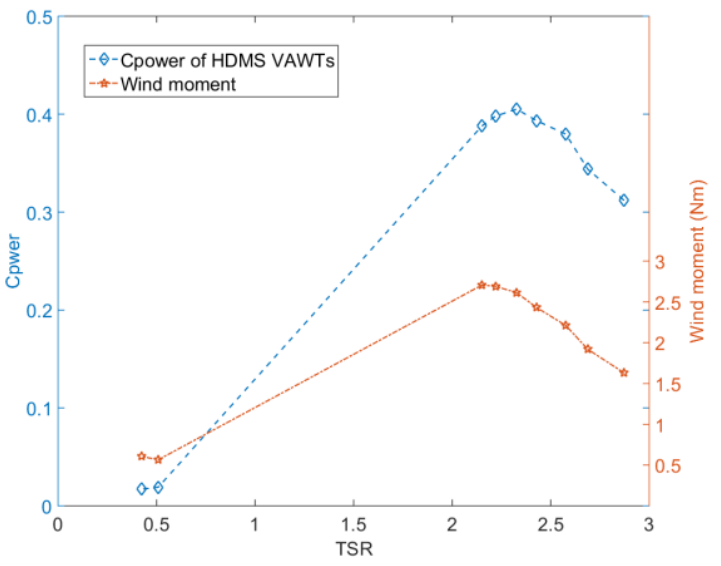

(b)

Figure 11. Power coefficient and wind moment as a function of TSR for (a) the Savonius VAWT and (b) the HDMS VAWT.

\section{III.C. HDMS VAWTs}

As mentioned previously, a linear damping effect is added to the VAWTs when the system reached a stable state under free loading conditions. The variation of TSRs as a function of the damping factor C for the HDMS VAWT is presented in Figure 10 (b). Similar to the Savonius VAWT, angular velocity of the HDMS VAWT decreases when the damping factor becomes lager. But at some certain value (in this case, $\mathrm{C}=0.1775$ ), the TSR would have a sharp decrease due to dynamic stall. As a result, the TSR would become aperiodic and unpredictable. The variation of power efficiency as a function of TSRs is displayed in Figure 11 (b). For the HDMS VAWT, the variation trend of moment is similar to that of the modified Savonius one. But when TSR decreases much, i.e., at damping factor $\mathrm{C}=0.1775$, the total aerodynamic moment would also decreases. It is observed from Figure 11 (b) that when the TSR 
approaches 2.3 , the power coefficient can reach its maximum value of approximately $40 \%$, which is about two thirds of the theoretical maximum value (59.3\%).

As has been recognized, dynamic stall can occur on Darrieus blades at large angles of attack (AOA) [16]. The histories of the angular velocity of two typical cases are shown in Figure 12 (a). One is the case when the power coefficient reaches its maximum value $40 \%$ ( $T S R=2.3$ ), the other one is when the damping factor $\mathrm{C}$ reaches 0.1775 , at which the movement of VAWTs becomes aperiodic and unpredictable (the mean TSR is 0.5 ). Figure 12 (b) and (c) show the vorticity fields in the $\mathrm{z}$ direction of those two cases. It is observed that comparing with the blade C, as shown in Figure 12 (b), when TSR is 2.3, larger flow separation occurs on top of the blade D, as shown in Figure 12 (c), when TSR is 0.5. In additional, the aerodynamic moment acting on the blade $\mathrm{C}$ is about $1.497 \mathrm{~N} \cdot \mathrm{m}$, while the aerodynamic moment acting on the blade $\mathrm{D}$ has a negative value about $-1.237 \mathrm{~N} \cdot \mathrm{m}$. Thus, dynamic stall has significant effect on the performance of the Darrieus blade. To explain these phenomena, the local AOA for the blade in the position of C and D is analyzed. In Figure 13, it is clearly seen that when TSR decreases, the local AOA experienced by the Darrieus blades will increase, resulting dynamic stall on the suction surface of the airfoil. As a result, when the TSR is small, regions with large flow separation will show up. This leads to a low aerodynamic moment. It also explains the differences of the vorticity fields as displayed in Figure 8 (b) and (c).

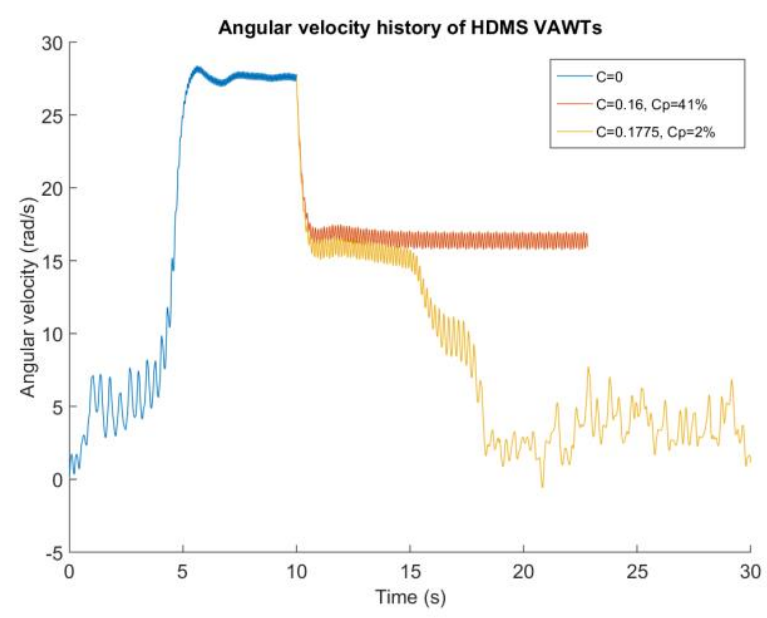

(a)

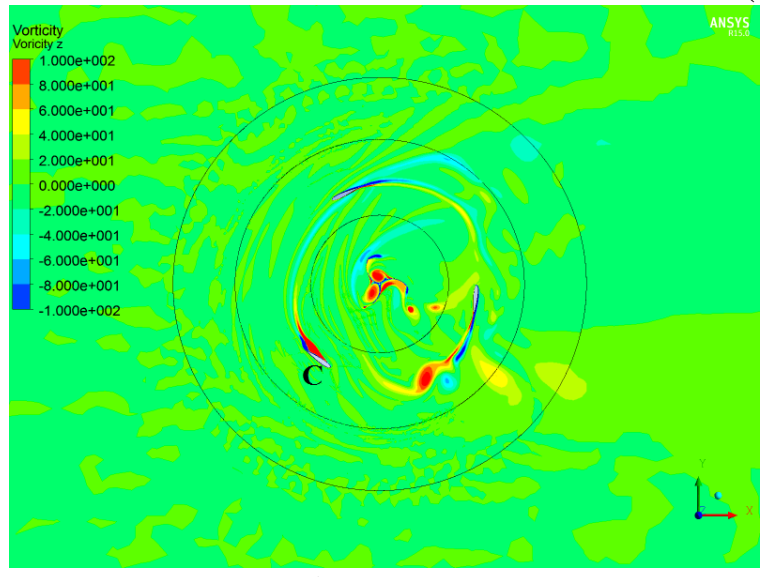

(b)

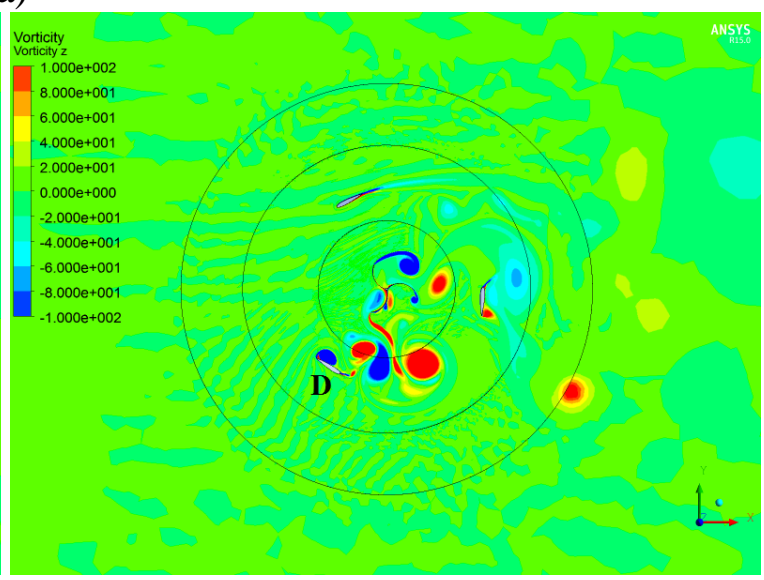

(c)

Figure 12. (a) Angular velocity history; (b) vorticity fields in the $\mathrm{z}$ direction when $C_{\text {power }}=40 \%$ (TSR=2.3); (b) and when $C_{\text {power }}=2 \%(\mathrm{TSR}=0.5)$. 


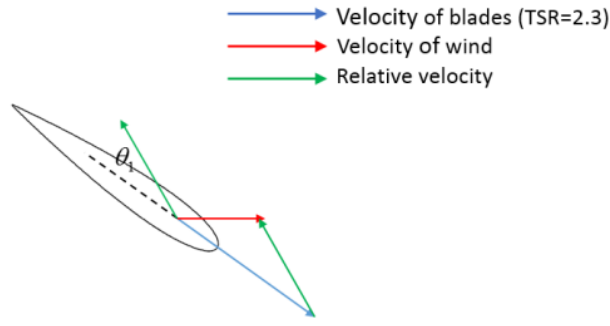

(a)

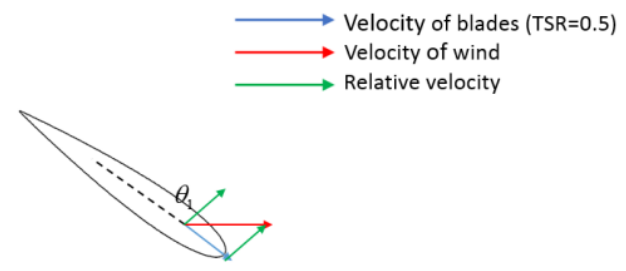

\section{Conclusions and future work}

In this study, unsteady flow physics of a modified Savonius turbine and an HDMS turbine are numerically studied using a fluid-structure interaction approach. From the results, it is found that for the modified Savonius VAWT, the best energy harvesting performance is achieved at small TSRs (i.e., around 1.2); while for the HDMS one, the best energy harvesting performance is achieved at a large TSR about 2.3. The maximum power coefficient of the HDMS VAWT is about $12 \%$ higher than that of the Savonius VAWT. However, under certain circumstances, i.e., the damping factor $\mathrm{C}$ reaches a certain value, the TSR and power coefficient of the HDMS VAWT can sharply descend to a low value due to the dynamic stall. Hence, when HDMS VAWTs are used in practical application, the inertia and loading of the turbine system should be designed properly based on the energy output requirement. In the future research, different geometric, dynamic and kinematic parameters will be simulated. These parameters include, but not limited to, VAWT blade sizes, incoming wind velocities, turbulence intensity, and wind gust effects.

\section{Acknowledgement}

The authors gratefully acknowledge the support of the Maryland Higher Education Commission through the Maryland Offshore Wind Energy Research Challenge Grant MOWER 14-03.

\section{References}

[1] Chao Li, Songye Zhu, You-lin Xu, Yiqing Xiao, "2.5D large eddy simulation of vertical axis wind turbine in consieration of high angle of attack flow," Renewable Energy, pp. 317-330, 2013.

[2] S. Eriksson, H. Bernhoff and M. Leijon, "Evaluation of different turbine concepts for wind power," Renewable and Sustainable Energy Reviews, vol. 12, pp. 1419-1434, 2008.

[3] M. M. A. Bhutta, N. Hayat, A. U. Farooq, Z. Ali, S. H. Jamil and Z. Hussain, "Vertical axis wind turbine - A review of various configurations and design techniques," Renewable and Sustainable Energy Reviews, vol. 16, pp. 1926-1939, 2012.

[4] D. MacPhee and A. Beyene, "Recent advances in rotor design of vertical axis wind turbines," Wind Engineering, vol. 36, pp. 647-666, 2012. 
[5] J. R. Baker, "Features to aid or enable self starting of fixed pitch low solidity vertical axis wind turbines," Journal of Wind Engineering and Industrial Aerodynamics, vol. 15, pp. 369-380, 1983.

[6] B. Kirke and L. Lazauskas, "Enhancing the performance of a vertical axis wind turbine using a simple variable pitch system," Wind Engineering, vol. 15, pp. 187-195, 1991.

[7] Y. Bazilevs, A. Korobenko, X. Deng, J. Yan, M. Kinzel and J. O. Dabiri, "Fluid-structure interaction modeling of vertical-axis wind turbines," Journal of Applied Mechanics, vol. 81, p. 081006, 2014.

[8] M. Nakajima, S. Iio and T. Ikeda, "Performance of double-step Savonius rotor for environmentally friendly hydraulic turbine," Journal of Fluid Science and Technology, vol. 2008, pp. 420-429, 2008.

[9] M. A. Kamojia, S. B. Kedarea and S. V. Prabhu, "Performance tests on helical Savonius rotors," Renewable Energy, vol. 34, pp. 521-529, 2009.

[10] Ivan Dobrev , Fawaz Massouh, "CFD and PIV investigation of unsteady flow through Savonius wind turbine," Energy Procedia, vol. 6, pp. 711-720, 2011.

[11] J. Gavalda, J. Massons and F. Diaz, "Experimental study on a self-adapting Darrieus-Savonius wind machine," Solar \& Wind Technology, vol. 7, pp. 457-461, 1990.

[12] Wakui Tetsuya, Yoshiaki Tanzawa, Toshio Nagao, "Hybrid configuration of Darrieus and Savonius rotors for stand-alone wind turbine-generator systems," Electrical Engineering in Japan, vol. 150, pp. 259-266, 2004.

[13] R. Gupta, A. Biswas and K. K. Sharma, "Comparative study of a three-bucket Savonius rotor with a combined three-bucket Savonius - three-bladed Darrieus rotor," Renewable Energy, vol. 33, pp. 1974-1981, 2008.

[14] Daniel B. Araya, John O. Dabiri, "A comparison of wake measurement in motor-driven and flowdriven turbine experiments," Experiments in Fluids, 2015.

[15] Maosheng Zheng, Yusheng Li, Yangyang Tian, Jun Hu, Yuan Zhao, Lijun Yu, "Effect of blade installation angle on power efficiency of resistance type VAWT by CFD study," International Journal of Energy and Environmental Engineering, vol. 6, no. 1, pp. 1-7, 2015.

[16] A-J. Buchner M.W.Lohry, L.Martinelli, J.Soria, A.J.Smits, "Dynamic stall in vertical axis windturbines:Comparing experiments," WindEng.Ind.Aerodyn, p. 163-171, 2015.

[17] A. Betz, Introduction to the Theory of Flow Machines., Oxford: Pergamon Press, 1966.

[18] K. W. MCLAREN, A NUMERICAL AND EXPERIMENTAL STUDY OF UNSTEADY LOADING OF HIGH SOLIDITY VERTICAL AXIS WIND TURBINES, McMaster University, 2011.

[19] N. N. Sørensen and J. A. Michelsen, "Drag Prediction for Blades at High Angle of Attack Using CFD," Sol. Energy Eng, vol. 126(4), pp. 1011-1016, 2004.

[20] A. Travin, M. Shur, M. Strelets, P. R. Spalart, "Physical and Numerical Upgrades in the DetachedEddy Simulation of Complex Turbulent Flows," Fluid Mechanics and Its Applications, pp. 239254, 2004.

[21] Dominy, R. and Lunt, P. and Bickerdyke, A. and Dominy, J., "Self-starting capability of a Darrieus turbine," I MECH E part A : journal of power and energy, vol. 1, no. 221, pp. 111-120, 2007. 
[22] David Wafula Wekesa, Cong Wang, Yingjie Wei, Weidong Zhu, "Experimental and numerical study of turbulence effect on aerodynamic performance of a small-scale vertical axis windturbine," Journal of Wind Engineering and Industrial Aerodynamics, vol. 157, p. 1-14, 2016. 